\title{
Mechanisms of Rhythm Generation in a Spinal Locomotor Network Deprived of Crossed Connections: The Lamprey Hemicord
}

\author{
Lorenzo Cangiano and Sten Grillner \\ Nobel Institute for Neurophysiology, Department of Neuroscience, Karolinska Institute, SE-17177 Stockholm, Sweden
}

The spinal network coordinating locomotion in the lamprey serves as a model system, in which it has been possible to elucidate connectivity and cellular mechanisms using the isolated spinal cord. Locomotor burst activity alternates between the left and right side of a segment through reciprocal inhibition. We have recently shown that the burst generation itself in a hemisegment does not require inhibitory mechanisms. The focus of this study is the intrinsic operation of this hemisegmental burst-generating component of the locomotor network.

Brief electrical stimulation $(0.3 \mathrm{~s})$ of the hemicord evokes long-lasting bouts ( $>2 \mathrm{~min})$ of bursts $(2-15 \mathrm{~Hz})$ in the mid to high-frequency range of locomotion. Bout release is an all-or-none phenomenon requiring a threshold intensity of stimulation and glutamatergic transmission within a population of excitatory interneurons, with axons extending over several segments. The progressive activitydependent decrease in burst frequency that takes place during a bout is followed by a slow recovery process lasting $>20$ min. Intracellular recordings of single motoneurons, excitatory interneurons, and inhibitory interneurons show that locomotor bouts, in general, are accompanied by a marked depolarization. Membrane potential oscillations and spikes occur in phase with the ventral root (VR) bursts. Active motoneurons and interneurons fire one spike per VR burst, as also confirmed by axonal recordings. Thus, the reciprocal inhibition between opposite hemisegments in the intact cord not only ensures left-right alternation and lowers the locomotor frequency but also promotes a shift from single to multiple action potentials per cycle in network neurons.

Key words: locomotion; central pattern generator; spinal; lamprey; rhythm; synchrony

\section{Introduction}

In lamprey, as in other vertebrates, locomotor movements are coordinated by a spinal network of interneurons referred to as a central pattern generator (CPG), which is activated from the brainstem. The intrinsic functioning of the vertebrate locomotor CPG has been studied in greater detail in the adult lamprey and the frog embryo than in other experimental models (Rossignol and Dubuc, 1994; Roberts et al., 1998; Earhart and Stein, 2000; Grillner et al., 2000; Sillar et al., 2002; Kiehn and Butt, 2003). At the segmental level, the spinal circuitry provides rhythmic excitation of motoneurons (MNs) on each side from ipsilateral glutamatergic interneurons and crossed inhibition from glycinergic interneurons to ensure alternation (Buchanan and Grillner, 1987; Grillner, 2003). The ability to generate this motor program is distributed along the spinal cord.

Received June 11, 2004; revised Dec. 2, 2004; accepted Dec. 4, 2004.

This work was supported by the Swedish Medical Research Council (VR-M 3026, VR-NT 1496), European Union Grant QLG3-CT-2001-01241, the M. and M. Wallenberg Foundation, the Karolinska Institute, and the Christopher Reeve Paralysis Foundation. L.C. is a recipient of a Marie Curie doctoral fellowship from the European Commission. We are grateful to Drs. A. Cangiano, A. El Manira, R. H. Hill, D. Schmitt, and P. Wallén for valuable comments on this manuscript. We also express our appreciation to M. Bredmyr for skillful laboratory assistance.

Correspondence should be addressed to Dr. Sten Grillner, Department of Neuroscience, Karolinska Institute, SE-17177 Stockholm, Sweden. E-mail: sten.grillner@neuro.ki.se.

D01:10.1523/JNEUROSCI.2301-04.2005

Copyright $\odot 2005$ Society for Neuroscience $\quad$ 0270-6474/05/250923-13\$15.00/0
The operation of the lamprey CPG has been investigated extensively using the isolated spinal cord preparation, with the motor pattern evoked typically by perfusion of glutamatergic agonists (Cohen and Wallén, 1980; Poon, 1980). The circuitry generating the motor rhythm is present in each of the two sides of the cord (Cangiano and Grillner, 2003), and crossed inhibitory connections ensure alternation but also downregulate the endogenous unilateral frequency (Grillner and Wallén, 1980; Cohen and Harris-Warrick, 1984; McPherson et al., 1994; Cangiano and Grillner, 2003). If the left and right hemicords are gradually separated by surgical means, the locomotor frequency increases until, when completely hemisected, they independently express fast rhythmic bursting. The hemicord can be activated either pharmacologically (NMDA, D-glutamate) or by electrical stimulation. Ipsilateral glycinergic inhibition is not essential for rhythm generation, and even single hemisegments produce rhythmic bursting. In NMDA, the hemicord can also express bursting in the slow locomotor range (Cangiano and Grillner, 2003).

The goal here is to investigate the operation of the unilateral burst-generating component of the locomotor CPG in the medium-fast swim frequencies, using the electrically activated hemicord. We first explore the dynamics of ipsilateral excitatory interaction and then dissect the firing pattern of single neurons in relation to the locomotor bursting. This method with brief reproducible stimuli, which is ineffective when applied to the intact 
spinal cord (Fagerstedt et al., 2000), is advantageous over perfusing glutamatergic agonists. Intracellularly recorded neurons can be identified at rest, and well organized locomotor bursting can be induced at once. After stimulation, the hemicord expresses a bout of locomotor activity lasting several minutes, by its endogenous synaptic interactions.

We report that at the core of the hemicord network lies a population of glutamatergic interneurons that can sustain longlasting activity, requiring fast synaptic interaction. Slow modulation via, for example, metabotropic glutamate receptors may also contribute to the overall depolarizing drive. An activitydependent mechanism reduces network excitability during a bout, which recovers when the network is silent. Within a bout, interneurons and MNs fire one action potential (or less) for every locomotor cycle, partially synchronizing with each other. Given that in the intact spinal cord neurons can fire multiple spikes per cycle, crossed inhibition appears to also play a role in the patterning of single neuron activity.

\section{Materials and Methods}

A total of 45 adult lampreys (Lampetra fluviatilis) were anesthetized by immersion in tricaine methane sulfonate (MS-222, $200 \mathrm{mg} / \mathrm{L}$; Sigma, St. Louis, MO) and decapitated caudal to the gills. The spinal cord preparations (between 8 and 15 segments, except when stated otherwise) were dissected from the region between the gills and the first dorsal fin, together with the dorsal half of the notochord as a mechanical support. After pinning down these preparations in a Sylgard-lined chamber, the meninges were peeled off from the dorsal surface of the cord. During the dissection and thereafter, preparations were perfused with cooled physiological solution $\left(5-10^{\circ} \mathrm{C}\right)$. The Ringer's solution [containing (in $\mathrm{mm}$ ) $138 \mathrm{NaCl}, 2.1 \mathrm{KCl}, 1.8 \mathrm{CaCl}_{2}, 1.2 \mathrm{MgCl}_{2}$, 4 glucose, 2 HEPES, and 0.5 L-glutamine] was bubbled with $\mathrm{O}_{2}$ and adjusted to $\mathrm{pH} 7.4$ with $\mathrm{NaOH}$. Hemicords were obtained by cutting along the midline using a Micro Feather ophthalmic scalpel with a narrow tip $\left(15^{\circ}\right.$ model; Feather Safety Razor, Osaka, Japan), as described by Cangiano and Grillner (2003).

Electrophysiology. The hemicords were activated by delivering a brief train of voltage pulses $(n=10$, except when stated otherwise; $2 \mathrm{~ms}$ duration, $33 \mathrm{~Hz}$ ) to the dorsal surface of the preparation with a coarse glass pipette electrode (see Fig. $1 \mathrm{~A}$ ). A small gap was kept between the pipette tip and cord surface to avoid any mechanical damage to the tissue (see Fig. $1 A$, inset). The position of this electrode and the voltage level were never altered during an experiment. Except where stated differently, the pulse voltage was set well above the threshold for eliciting a locomotor bout $(4-8 \times)$. Ventral root (VR) activity was recorded with similar glass pipette electrodes juxtaposed to the VR stump. Signals were sent to a differential AC amplifier (model 1700; A-M Systems, Everett, WA) and bandpass filtered between 100 and $500 \mathrm{~Hz}$. The output from the amplifier was acquired at a sampling rate of $2 \mathrm{kHz}$ (or higher) per channel on a personal computer equipped with an analog-to-digital converter (Digidata 1320A) and running Clampex 8 software (both from Axon Instruments, Union City, CA). Single and paired intracellular recordings were made with sharp electrodes pulled to a final resistance of $80-120 \mathrm{M} \Omega$, filled with $3 \mathrm{~m}$ potassium acetate and $0.1 \mathrm{M} \mathrm{KCl}$. Intracellular signals were fed to an Axoclamp 2A amplifier (Axon Instruments) working in bridge mode, low-pass filtered at $1 \mathrm{kHz}$, and further amplified. The signals were then acquired at a sampling rate of $6.7 \mathrm{kHz}$ per channel on the system described above. In some experiments, single propriospinal axons were recorded extracellularly using sharp electrodes filled with $3 \mathrm{M} \mathrm{NaCl}$ (10-15 M 2 ). To facilitate penetration into the tissue, $0.5 \mathrm{mg} / \mathrm{ml}$ collagenase (Sigma) was pre-perfused for $5 \mathrm{~min}$. The signal from the electrode was sent to the same amplifier used for the VR recordings and acquired at $2 \mathrm{kHz}$. All micropipettes were pulled from thin-walled aluminosilicate glass (Sutter Instrument, Novato, CA). To improve tissue stability during intracellular recordings, a tiny strip of Sylgard was inserted between the opposite hemicords at the level of the microelectrode.

Identification of neuronal type. MNs were identified, as in previous work, by recording the extracellular spike in the VR (Cangiano et al.,
2002). Excitatory premotor interneurons were identified either by paired intracellular recording with a postsynaptic $\mathrm{MN}(n=3$; EPSP amplitude, $0.34 \pm 0.11 \mathrm{mV})($ see Fig. $6 \mathrm{~A}$ ) or by detecting with an extracellular electrode the summed EPSPs evoked by them in MNs and electrotonically propagated into a VR $(n=3$; EPSP amplitude, $0.62 \pm 0.46 \mu \mathrm{V})$ (see Fig. $6 B_{1}$ ). In this technique (Brink et al., 1981; Butt and Kiehn, 2003), the cutoff of the high-pass filter normally used in the VR recordings (AC recording) was reduced to $0.1 \mathrm{~Hz}$ (quasi-DC recording), and spiketriggered averaging of a few hundred sweeps was performed. Inhibitory premotor interneurons were identified either by DC VR recording of the propagated IPSPs $(n=2$; IPSP amplitude, $-0.45 \pm 0.26 \mu \mathrm{V})$ (see Fig. $6 B_{2}$ ) or by a reduction of the efferent VR activity (AC recording) in the activated hemicord, during high-frequency trains of action potentials in the inhibitory neuron $(n=3 ; 20-50$ spikes at $40 \mathrm{~Hz}$ ) (see Fig. $6 C$ ). Note that the use of averaging to reveal excitatory and inhibitory synaptic potentials in MNs may identify interneurons mediating their effect polysynaptically. With the inhibitory premotor interneuron, we thus refer to its final effect on MNs.

Pharmacology. When testing the effect of a drug on the hemicord, locomotor bouts were elicited every $10 \mathrm{~min}$ before and during drug perfusion. Drugs were dissolved in Ringer's solution, and the $\mathrm{pH}$ was adjusted to 7.4 with $\mathrm{NaOH}$. The flow rate of the perfusion system was kept constant throughout an experiment. CNQX disodium salt (Tocris, Ellisville, MO) was used to block AMPA and kainate receptors. D-AP-5 (Tocris) was used to block NMDA receptors. 5-Hydroxytryptamine (5HT) hydrochloride (Sigma-Aldrich, St. Louis, MO) was used at $1 \mu \mathrm{M}$. Split bath preparations were arranged, as in previous studies (Dale, 1986), with a thin plastic barrier sealed with petroleum jelly. The fluid level in the pool perfused with cadmium chloride $\left(\mathrm{CdCl}_{2} ; 200 \mu \mathrm{M}\right)$ was kept several millimeters below that of the other pool. The cadmium blockade of neurotransmitter-mediated synaptic transmission (Cangiano et al., 2002) was confirmed by the absence of any VR activity in the segments perfused with cadmium, despite a locomotor bout being evoked on the other side of the barrier.

Data analysis and statistics. Recordings were analyzed using custom scripts run within Axograph 4.9 software (Axon Instruments) on a Macintosh G5 computer (Apple Computer, Cupertino, CA). The cycle period (and thus burst frequency) and rhythmic quality were measured on the normalized autocorrelogram of the rectified VR recordings. The coefficient of rhythmicity was defined as the ratio $(\alpha-\beta) /(\alpha+\beta)$, where $\alpha$ and $\beta$ are the height of the second peak and the first trough in the autocorrelogram, respectively. This coefficient ranges between 0 and 1 , with higher values corresponding to stronger rhythmicity in the VR record (Cangiano and Grillner, 2003). The average amplitude of subthreshold membrane potential oscillations was quantified by the root mean square (RMS) (or SD) value taken over at least $15 \mathrm{~s}$ of recording, after having removed slow changes in membrane potential. The latter operation was done by subtracting a low-pass filtered copy of each record (running averaged with a window of $1 \mathrm{~s}$ ). Cross-correlation analysis of membrane potential oscillations versus VR bursting was performed in the following sequence. The VR trace was rectified and smoothed by a running average window $15 \mathrm{~ms}$ wide. A $15-75 \mathrm{~s}$ long stretch of recording was then subdivided in sections of $750 \mathrm{~ms}$ each. Cross-correlograms were computed for every section, and from the entire set, an average and SE were obtained (see Fig. $8 A_{2}, B_{2}$ ). Membrane potential oscillations were considered significantly correlated to VR bursting if the central peak of the average cross-correlogram exceeded four times the SE (Kremer and Lev-Tov, 1997). Cross-correlation analysis of action potentials versus VR bursting followed the same scheme, except that all non-spike data (subthreshold membrane potential fluctuations) were first removed from the raw intracellular records. All data in the text and figures are otherwise expressed as mean $\pm \mathrm{SD}$.

Numerical simulation. The theoretical relationship (see Fig. $1 F$, dashed curves) between efferent activity and burst frequency, given a constant number of spikes per VR burst (M), was determined empirically, as follows: a representative extracellularly recorded action potential was obtained by extracting from the VR record many spikes occurring in isolation, aligning and averaging them together. This spike template was used, in a computer routine, to generate simulated records of VR burst 

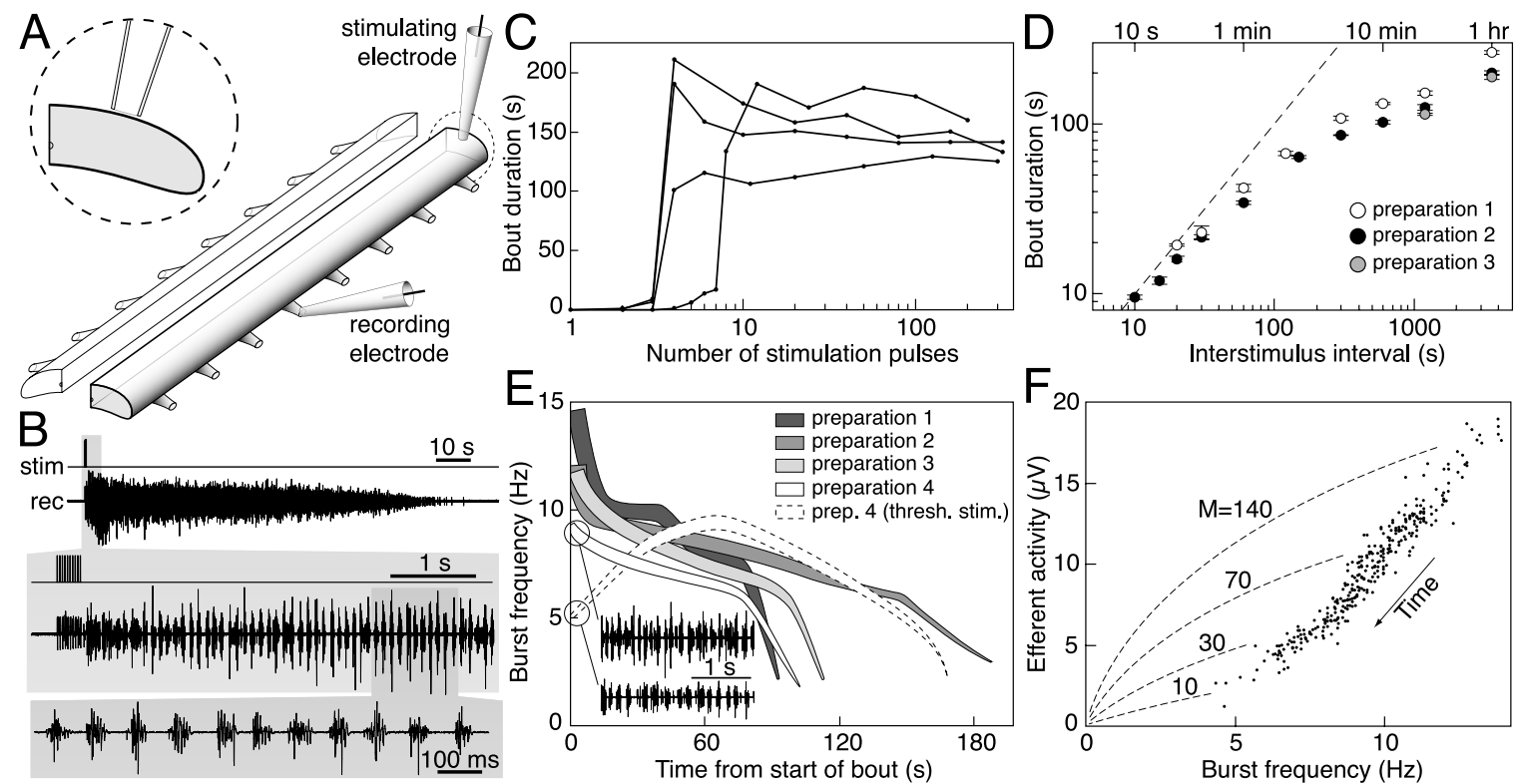

Figure 1. General properties of the motor response evoked by electrical stimulation in the lamprey hemicord. $A$, Hemicord preparation rigged for electrical stimulation and recording of VR activity with pipette electrodes (notochord not drawn). B, A brief train of pulses delivered via the stimulating electrode (stim) evokes a long bout of motor bursting at the VRs [recording (rec)] as first described by Cangiano and Grillner (2003). C, Duration of the VR response evoked in four preparations by stimulus trains containing an increasing number of pulses. A sharp threshold separates short responses from full-blown bouts. $D$, Effect of varying interstimulus interval on bout duration in three preparations (note the log scale on both axes). The longer intervals were tested before the shorter ones. Error bars indicate SD, and the dashed line marks where bout duration would be equal to the interstimulus interval. $E$, Time course of burst frequency (silhouette height) and efferent activity (width perpendicular to the silhouette axis) during a bout in four preparations. In one preparation, the stimulation intensity was also reduced to a level barely above the threshold for eliciting a bout, and in this case, a slow buildup of network activity occurred (dashed, open silhouette; compare with the continuous open one). thresh. stim., Threshold stimulation. F, Relationship between level of efferent activity (average of rectified VR trace) and burst frequency during the course of a bout (time flows from top right to bottom left). The dashed curves represent the theoretical trajectories (see Materials and Methods) that would be followed if M remained constant throughout a bout (see Results). Given that MNs fire, at most, once for every cycle (see Figs. 4,9), the steep trend in the actual data points shows that MNs are progressively derecruited during a bout.

activity with a duty cycle (i.e., burst proportion) of $50 \%$. For every particular burst frequency $(f)$ and value of M, a fictive VR record of $1 \mathrm{~s}$ was generated by adding at random positions within the bursts $(f \times M$ spike templates). Finally, each record was rectified and averaged to obtain the level of efferent activity indicated by the dashed curves in Figure $1 \mathrm{~F}$.

\section{Results}

As first described by Cangiano and Grillner (2003), the isolated hemicord is normally quiescent, but it exhibits a long-lasting bout (several minutes) of VR activity when electrically stimulated with a brief $(<1 \mathrm{~s})$ train of pulses (Fig. $1 A, B)$. This activity is organized into rhythmic bursts and was shown to represent the locomotor rhythm of fictive swimming in the absence of crossed inhibition (Cangiano and Grillner, 2003). We found this to be a robust network behavior, expressed in all hemicord preparations tested $(n=63)$.

\section{The release of a bout is an all-or-none phenomenon}

In the hemicord, a locomotor bout persists long after the stimulus that evoked it has ceased (Cangiano and Grillner, 2003). Is this a graded or an all-or-none response? In other words, does the bout duration depend on the "amount" of stimulation provided? To investigate this issue, we delivered, with several minutes interval, trains of $\mathrm{N}$ pulses ( $2 \mathrm{~ms}$ pulse duration, $33 \mathrm{~Hz}$ ) with $\mathrm{N}$ increasing from 1 to $\sim 300$. Pulse voltage was set to a threshold level at which, when a single pulse was delivered to the hemicord, a few spikes began to appear in the VR record. Figure $1 C$ shows results from four preparations displaying a marked nonlinearity in the response to this protocol. When a threshold number of pulses was reached, bout duration increased from a few seconds to a few minutes. Past this point up to several hundred pulses in a stimulus train, bout duration remained relatively constant. The sharp threshold-based onset of a regenerative bout, together with its long-lasting duration after the end of the stimulus, may be explained by the existence of a positive feedback between excitatory interneurons (EINs) in the hemicord locomotor circuitry. A similar mechanism has been suggested to also operate in other spinal systems (Roberts and Perrins, 1995; Tabak et al., 2000).

\section{Bout duration depends on the recent history of activity}

The more frequently a hemicord is stimulated, the shorter the duration of the bouts. This was examined by measuring bout duration as a function of the interstimulus interval. Several samples were taken in succession for each particular interval, starting from the longest, $60 \mathrm{~min}$, down to $10 \mathrm{~s}$. In these and most of the following experiments, the stimulus train consisted of 10 pulses at $33 \mathrm{~Hz}$, with a constant pulse amplitude. Figure $1 D$ shows that bout duration is strongly dependent on the interstimulus interval ( $n=3$; note the log scales). Under a 10-20 s interval, VR activity was still present when the next stimulus was delivered. The trialto-trial variability in the duration of the bouts at any given interval was quite small [average coefficient of variation (CV), $0.03 \pm$ $0.02]$. Another finding was the significant decrease in bout duration observed between 60 and $20 \mathrm{~min}(-38,-42$, and $-40 \%$, respectively; $p<0.01$; Wilcoxon-Mann-Whitney test). The unilateral locomotor network thus requires $>20$ min to fully recover in excitability.

Burst frequency and efferent activity decrease over the course of a bout

Within a bout, VR activity is organized in rhythmic bursts (Fig. $1 B)$. These we have previously shown to be directly related to the locomotor bursting expressed by the intact spinal cord during 
fictive swimming (Cangiano and Grillner, 2003). The frequency of bursting was highest immediately after the electrical stimulation, typically in the range of $10-15 \mathrm{~Hz}$, and decreased progressively, alongside a diminishing amount of efferent activity, down to $2-3 \mathrm{~Hz}$ (Fig. $1 E$, silhouettes). The relative level of efferent activity during a bout is represented by the width of the silhouettes (Fig. $1 E$ ) and was measured by the average amplitude of the rectified VR trace. If stimulus strength was decreased to the point of being barely on the threshold for eliciting a bout, burst frequency and efferent activity were initially low, and up to $20-50 \mathrm{~s}$ were required $(n=$ 5 ) for them to reach their maximum in the bout (Fig. 1E, compare dashed open silhouette, continuous open silhouette). This slow time course, which presumably represents the buildup of excitation within the isolated locomotor network, stands in striking contrast to the speed with which organized bursting is produced after a sufficiently strong stimulus (for an example, see Fig. $1 B$ ).

\section{Burst frequency and level of efferent activity are strongly linked}

In Figure $1 E$, burst frequency and the level of efferent activity appeared to vary in parallel. We further explored how they relate to each other in graphs as illustrated in Figure $1 F$ (one preparation shown). The full extent of a bout was subdivided in consecutive bins of $2.5 \mathrm{~s}$ duration (an interval several times longer than the maximum cycle period), each of which was used to determine one data point. Data from several consecutive bouts (elicited at a constant interval) contribute to the graph. Efferent activity is measured by the average amplitude during each bin of the rectified VR trace (minus the value obtained from a reference bin with only baseline noise). The two variables, frequency and level of efferent activity, follow, with time, a well defined consensual relationship from the top right to the bottom left of the graph (Fig. $1 F$ ). This trend appeared in all preparations in which such an analysis was performed $(n=20)$ and can be easily interpreted to represent a progressive derecruitment of $\mathrm{MNs}$ as the burst frequency decreases. This is illustrated by the theoretically derived curves described below (Fig. $1 F$, dashed curves $=140-10$ ) (see also figure legend). Should MN spikes have negligible duration and thus not interfere with each other, efferent activity would be a linear function of the number of spikes per second [i.e., of burst frequency given a constant number of spikes per burst (M)]. In reality, extracellularly recorded spikes have finite duration and, being biphasic, may not only summate but also partially cancel out when overlapping. The relationship between efferent activity and burst frequency for any given value of $\mathrm{M}$ will thus be sublinear. We determined these sublinear relationships by numerical simulation (see Materials and Methods) for several values of M (Fig. $1 F$, dashed curves). We may now interpret any deviation of the real data points below each local theoretical curve as a decrease in the number of spikes per VR burst. Because MNs fire only once per cycle (see below), Figure $1 \mathrm{~F}$ shows that there is a progressive derecruitment of MNs within a hemisegment.

\section{Fast glutamatergic excitation is required for locomotor bout expression}

Given that locomotor bout activity depends on recurrent excitation within a network of interneurons (cf. Parker and Grillner, 2000), we examined the relative contribution of the AMPA/kainate and NMDA receptors. Their respective blockers CNQX $(n=$ $5)$ and D-AP-5 $(n=3)$ were applied separately. Figure $2 A$ (top) shows the effect of CNQX on efferent VR activity during a locomotor bout. Records were rectified, a running average with a time window of $1 \mathrm{~s}$ was applied, and, where possible, several consecutive bouts were averaged together. A partial AMPA/kainate blockade with $1 \mu \mathrm{M}$ CNQX reduced the peak burst frequency $(p<0.01)$ as well as the peak VR activity and overall bout duration ( $p<0.05$; Wilcoxon-Mann-Whitney test), whereas a full blockade with 10-35 $\mu \mathrm{M}$ CNQX (Alford and Grillner, 1990) abolished VR activity altogether $(n=5)$. To exclude that CNQX might have simply raised the stimulation threshold required for the release of a bout, we increased the stimulus strength $(n=4)$ from 10 pulses at $33 \mathrm{~Hz}$ up to 400 pulses at $67 \mathrm{~Hz}$ (also increasing pulse amplitude). The response was a brief episode of intense VR activity with a similar amplitude as the control response, but of drastically shorter duration ( $-90 \pm 5 \%$ change). In four preparations, we could compare rhythmic quality in control and dur- 
ing partial CNQX wash-in, using the coefficient of rhythmicity (see Materials and Methods). Rhythmic quality decreased significantly in each preparation ( $p<0.01$; Wilcoxon-Mann-Whitney test). Thus, AMPA/kainate receptors are required for the expression of long-lasting locomotor bouts in the hemicord and appear to be important for rhythm generation (Fig. $2 \mathrm{~A}$, middle traces). A full NMDA receptor blockade with $100 \mu \mathrm{M}$ D-AP-5 (Alford and Grillner, 1990) decreased the peak burst frequency $(p<0.01)$ and produced a significant $(p<0.01$; WilcoxonMann-Whitney test) but incomplete reduction in peak VR activity and bout duration in each case ( $-62 \pm 25 \%$ change) (Fig. $2 B$, top). Rhythmic quality during the bouts was unchanged in one preparation $(p=0.54)$, whereas it increased significantly in another two $(p<0.01)$. Thus, NMDA receptors contribute to the overall excitability during a bout but are not required for fast rhythm generation (Fig. $2 \mathrm{~B}$, middle traces) (cf. Brodin and Grillner, 1986a). Note that the observed reduction in peak VR activity and bout duration could, in principle, be attributable entirely to the antagonists acting at the synapse between EINs and MNs. The decrease in peak burst rates shows, however, that CNQX and D-AP-5 act within the rhythm-generating network itself.

We also plotted efferent activity versus burst frequency in control conditions and during a partial receptor blockade, either by perfusion of $1 \mu \mathrm{M} C N Q X$ (Fig. $2 A$, bottom plot) or by incomplete wash-in of $100 \mu \mathrm{M}$ D-AP-5 (Fig. $2 B$, bottom plot). The two variables clearly follow the same relationship, except that with a partial glutamatergic blockade, the response after stimulation is equivalent to that in control around mid-bout (arrows mark bout start). The activity-dependent mechanism terminating locomotor bouts could then involve a progressive reduction in the efficacy of fast glutamatergic synaptic transmission.

In addition to fast glutamatergic transmission, slower metabotropic components are likely to contribute in maintaining excitation from cycle to cycle during the bouts (Krieger et al., 1998; Kettunen et al., 2003).

\section{Longitudinal extension of the network influences bout duration}

In a similar manner to the pharmacological blockade of fast glutamate transmission, reducing the number of interneurons in the network would be expected to decrease bout duration. This can be obtained by varying the number of hemisegments in relation to the rostrocaudal projection distance of the relevant interneurons. We monitored bout duration at progressively smaller numbers of hemisegments by removing portions at the rostral and caudal ends $(n=2)$ (Fig. $3 A)$. Both the recording and stimulating electrodes were placed in the middle of the preparation and left undisturbed throughout an experiment. Bouts were elicited every $10 \mathrm{~min}$, and several recordings were made for each hemicord length until a stable time course was reached. Peak efferent activity and bout duration shortened progressively with the decreasing number of hemisegments ( $p<0.01$ for reductions of five or more hemisegments; Wilcoxon-Mann-Whitney test), from the longest to the shortest piece (Fig. $3 B, C$ ). The average decrease in bout duration in each preparation was -7 and $-5 \%$ per segment, respectively. EINs (Buchanan and Grillner, 1987) may reach postsynaptic targets as far as eight segments caudally and three segments rostrally (Dale, 1986). It is thus reasonable that, although a single hemisegment is sufficient to generate short bouts of organized bursting (Cangiano and Grillner, 2003), the interaction between many spinal segments can support a prolonged activity.

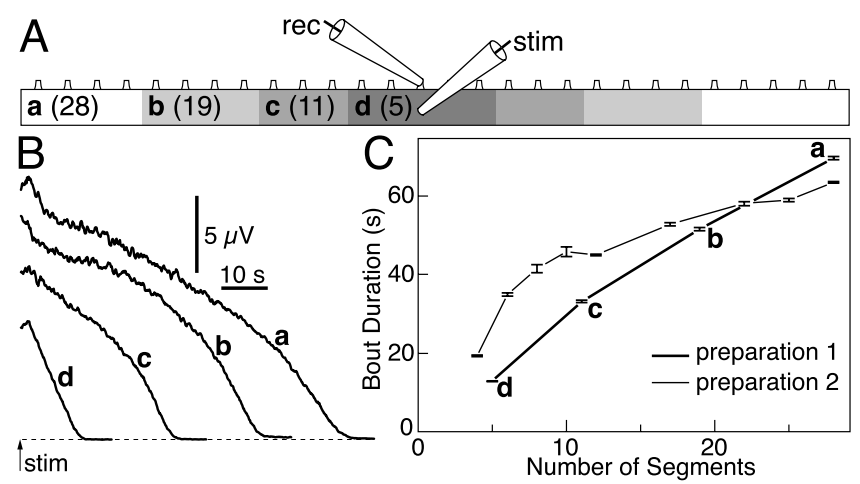

Figure 3. Bout duration decreases as the number of hemisegments is reduced. $A$, Recording (rec) and stimulating (stim) electrodes were placed in the middle of a 28-hemisegment-long preparation. Rostral and caudal portions were progressively removed to shorten the hemicord, with several bouts being evoked every $10 \mathrm{~min}$ at each particular length $(28,19,11$, and 5 hemisegments). $B$, Time course of efferent activity (running average of rectified VR trace) at the different lengths. Peak activity and bout duration decreased the shorter the preparation. $C$, Summary of bout duration as a function of number of hemisegments in two different preparations. Error bars indicate SE.

\section{MNs fire one spike per locomotor cycle}

What is the pattern of activity of single neurons in the active hemicord? We first examined MNs. Typically this has been done in the intact lamprey spinal cord by recording with sharp electrodes from the cell soma during fictive swimming (Buchanan and Cohen, 1982; Russell and Wallén, 1983; Buchanan and Kasicki, 1995). Concerns over the use of this technique have been raised, in that the damage inflicted to the membrane by the micropipette might modify the natural firing pattern of a neuron (Dale, 1995; Aiken et al., 2003). To avoid this problem, we recorded intracellularly from single motor axons $(n=7)$ in the VR (Fig. 4A), which are 2-20 $\mu \mathrm{m}$ thick (Rovainen and Dill, 1984). In this configuration, given the length and thinness of the axon, the site of impalement is electrotonically distant from the soma. After electrical stimulation of the hemicord, the $\mathrm{MN}$ axons, quiescent at rest, became active (Fig. $4 \mathrm{~B}$ ) throughout most of the locomotor bout (79 $\pm 19 \%$ of total bout duration). Spike frequency was initially high $(15.6 \pm 2.8 \mathrm{~Hz})$ and decreased progressively during the course of a bout. Except for this slow drift in the mean firing rate, activity was essentially regular as shown by the $\mathrm{CV}$ of interspike intervals $\left(\mathrm{CV}_{\text {ISI }}\right)$ measured [e.g., between 10 and $20 \mathrm{~s}$ from bout start $\left.\left(\mathrm{CV}_{\text {ISI }}=0.21 \pm 0.03\right)\right]$. MNs contributed with a single spike to every VR burst (Fig. 4B). This is summarized by a graph of individual interspike intervals (ISIs) in one axon and the cycle period of VR bursting measured over consecutive bins of $5 \mathrm{~s}$ (Fig. $4 C$ ). The two data sets overlap during the course of the bout (the missing data points for cycle period at the end of the bout are attributable to the onset of irregular bursting). In general, toward the end of a bout and just before returning silent, MNs skipped one or more cycle periods (data not shown). Data from all axons are summarized in a concise form in Figure $4 D$. The histograms show the distribution of ISIs with each interval normalized to the locomotor cycle period (each histogram was computed using the intervals falling in the first $66 \%$ of the axonal spike discharge during a bout, the most active part). The normalized ISIs form a single peak centered around a unit value, in agreement with the conclusion that MNs under these conditions fire one action potential for every VR burst. If one identifies the occurrence of multiple spikes in a cycle with a normalized ISI smaller than 0.5 , this was a very rare event (for the seven motor axons, in percentage of all normalized ISIs: $1.6 \%$ in the most active two-thirds of 
each spike discharge and $0.4 \%$ in the remaining one-third). The results from intrasomatic recordings of MNs are discussed further below (see Fig. 9).

Ipsilaterally projecting interneurons fire one spike per locomotor cycle

As for MNs, past studies looking at the pattern of activity of ipsilaterally projecting interneurons, the EINs, the small inhibitory ones (Buchanan and Grillner, 1988), and the lateral interneurons (Rovainen, 1974) are based on intracellular recordings with sharp electrodes (Buchanan and Cohen, 1982; Buchanan and Grillner, 1987, 1988; Buchanan et al., 1989). The likelihood of the micropipette modifying the electrical properties of the cell, and thus its firing pattern, is not negligible given the small size of these cells (but see Li et al., 2004). We therefore obtained extracellular recordings of single axons from ipsilaterally projecting neurons (contralateral side removed). A tight plastic/petroleum jelly barrier was built around the hemicord creating two compartments $(\sim 15$ and $\sim 3$ segments long, respectively), each with its own bath (Fig. $5 A)$. The larger pool $(\sim 15$ segments) was perfused with normal Ringer's solution, and the hemicord was rigged in the usual way to elicit/record locomotion. In the smaller pool (approximately three segments), synaptic transmission was blocked with $200 \mu \mathrm{M} \mathrm{CdCl}_{2}$, and the hemicord was penetrated with a sharp extracellular microelectrode, one to three segments from the barrier. Any spikes detected by this electrode must have originated from a neuron located in the other pool and extending an ipsilateral axon beyond the barrier. Several axons $(n=8)$ were found that, initially quiescent, discharged a long train of action potentials when a locomotor bout was elicited (Fig. $5 B)$. Caudally $(n=6)$ as well as rostrally $(n=2)$, projecting axons were recorded. These axons were relatively difficult to find, and their signal was lost if the microelectrode was moved by $>5-10$ $\mu \mathrm{m}$. The duration of discharge relative to the length of the locomotor bout was more varied among the interneurons ( $75 \pm 33 \%$ of total bout duration) than the MNs. In seven of eight axons, the spike frequency was initially high $(12.3 \pm 2.6 \mathrm{~Hz})$ and decreased during the bout, whereas in one axon, it began low and reached a peak (4.5 $\mathrm{Hz}$ ) around mid-bout. Spiking within the trains was rather regular $\left(\mathrm{CV}_{\text {ISI }}=0.23 \pm\right.$ 0.08 , measured in the most active $5 \mathrm{~s}$ of the discharge). The plot of Figure $5 C$ shows how, over the course of a bout, the ISIs of an axon clearly overlap the average cycle period of VR bursting (the missing data

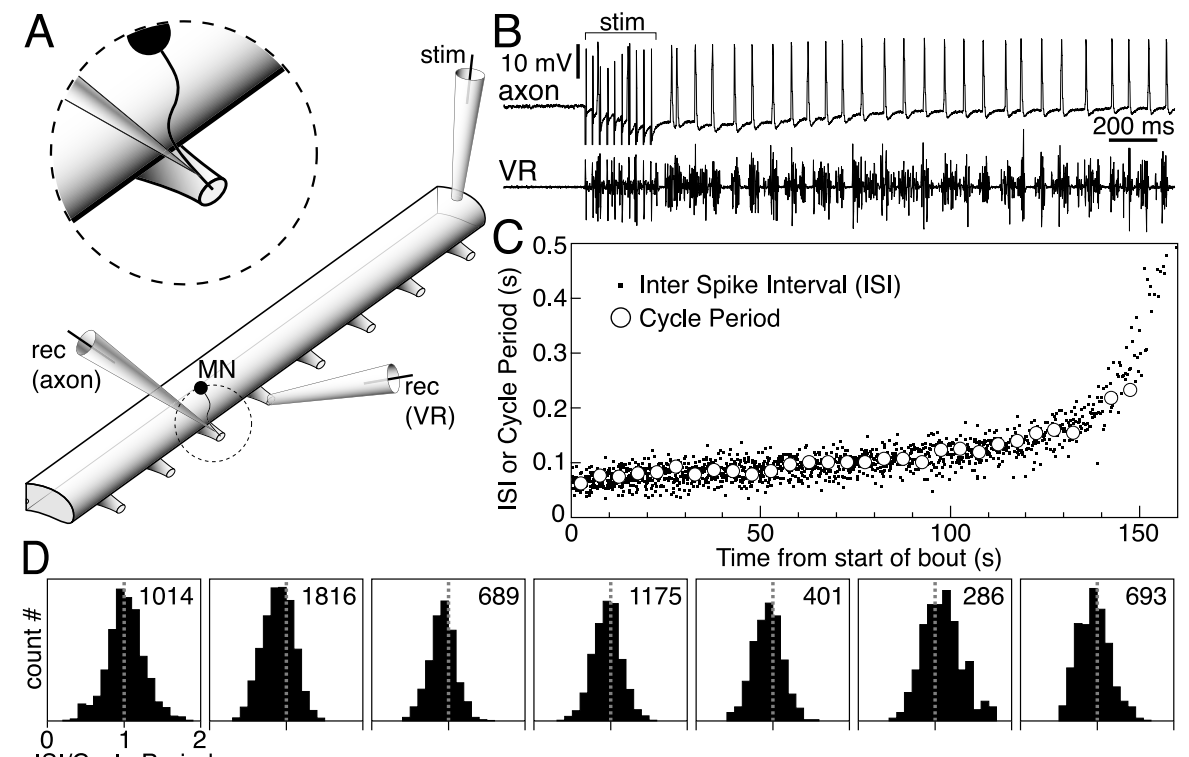

ISI/Cycle Period

Figure 4. MNs fire one action potential for every locomotor cycle. $A$, Intracellular recordings (rec) from single axons in a VR allowed study of the firing pattern of MNs without affecting the soma. $B$, When a bout was evoked by electrical stimulation (stim), regular spiking began in the axon. $C$, Plot showing both ISI in the axon and average cycle period of VR bursting during a bout. Because the two sets of data points overlap, it can be inferred that this MN fired once per cycle. $D$, Histograms summarizing the data from seven motor axons. For each axon, the information provided by a graph of the type shown in ( was condensed by normalizing all ISIs to the corresponding cycle period, counting their occurrences, and displaying them in histograms. Normalized ISIs form peaks centered on unity, which corresponds to a firing pattern of one spike per cycle. For clarity, a normalized ISI value of 0.5 means two spikes per cycle, whereas a value of 2 means one spike every second cycle. The number at the top right of each panel the number of ISls used in generating each histogram.

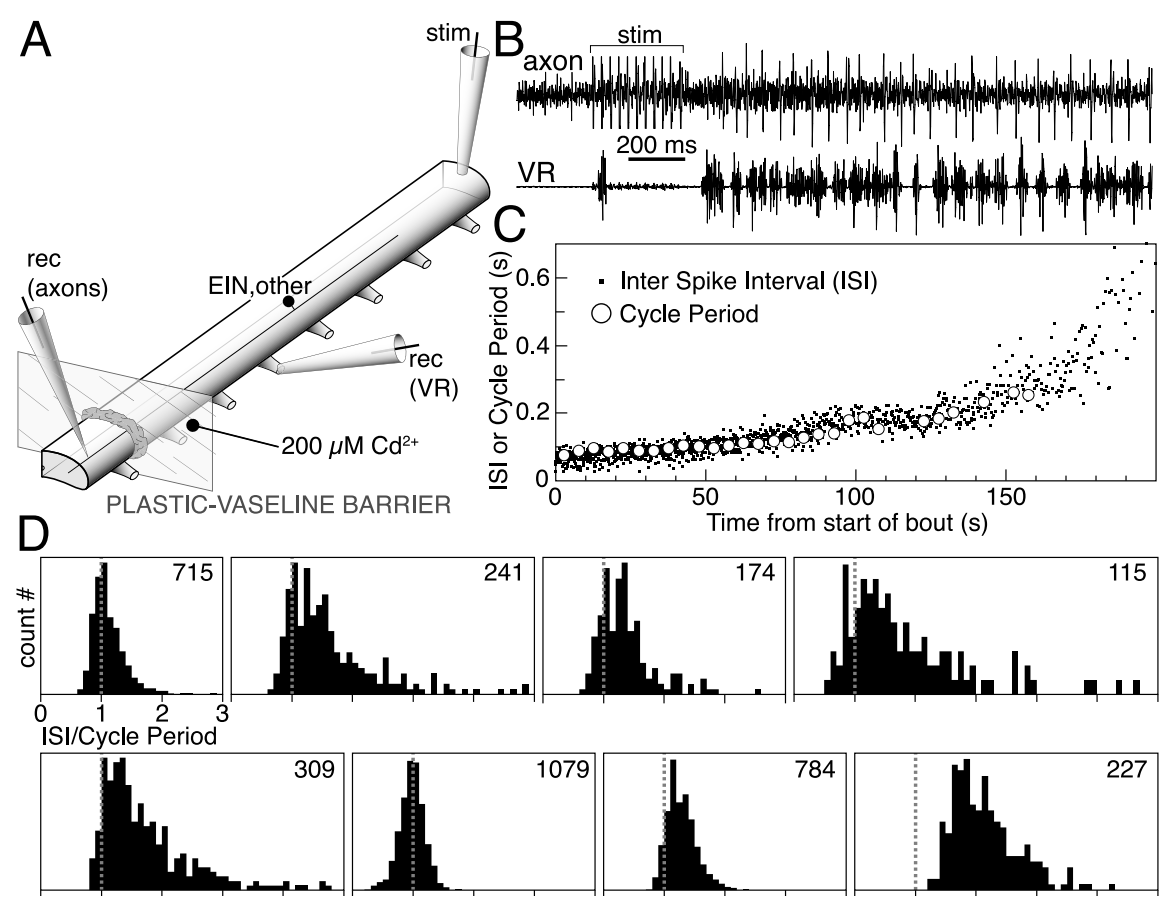

Figure 5. Ipsilaterally projecting interneurons fire one action potential for every locomotor cycle or less. $A$, Extracellular recordings (rec) from single axons of ipsilaterally projecting interneurons were made with a microelectrode inserted into the ventral hemicord, while evoking locomotor bouts. The identification of these neurons as ipsilaterally projecting was ensured by recording in a caudal or rostral section of the hemicord, separately perfused with $\mathrm{CdCl}_{2}$ to block all synaptic transmission. Any action potentials detected by the microelectrode must have originated from a soma located beyond the dividing barrier, and thus more than one segment away. $B$, Example of an axon in which regular firing began immediately after the induction of a bout by electrical stimulation (stim). C, Plot showing both ISI in the axon and average cycle period of VR bursting during a bout. Because the two sets of data points overlap, this interneuron fired once per cycle. D, Histograms summarizing the data from eight interneurons. In each case, all ISIs were normalized to the corresponding cycle period. Note that one axon fired on every other cycle (last histogram) and that all axons could skip one or more cycles. The number at the top right of each panel indicates the number of ISIs used in generating each histogram. 
points for the cycle period at the end of the bout are attributable to the onset of irregular bursting). Data from all axons is summarized in Figure $5 D$ in the same way as for MNs. In the most active part of the axonal discharge, a single interneuron spike was typically observed for every locomotor cycle (histogram peak centered on unity). In one axon, there was a tendency to skip every other cycle (histogram peak centered on 2). The histogram tails extending toward multiples of the cycle period indicate that when activity decreased, interneurons could skip one or more cycles. Note that these histograms are broader than if, when normalizing, we could take into account the cycle-to-cycle fluctuations in the cycle period. If one identifies the occurrence of multiple spikes in a cycle with a normalized ISI smaller than 0.5 , this was an extremely rare event (for the eight axons, in percentage of all normalized ISIs: $0.6 \%$ in the most active two-thirds of each spike discharge and none in the remaining one-third). The identity of these neurons could not be established directly, but they must belong to neurons with ipsilateral axons. The majority of the ipsilaterally projecting interneurons are thought to be excitatory (Buchanan and Grillner, 1988).

\section{Intracellular somatic recordings in the hemicord}

The electrically activated hemicord is an ideal preparation in that neurons can be observed in complete quiescence or during locomotion within a short time. We performed intracellular somatic recordings ( $n=65$, from 29 animals) of identified MNs $(n=29)$, excitatory $(n=6)$ or inhibitory $(n=4)$ premotor interneurons, as well as unidentified neurons $(n=26)$ that are unlikely to have comprised MNs, because no orthodromic spike was detected at the nearest VR. Interneurons were identified by observing excitatory or inhibitory synaptic potentials recorded in the postsynaptic neuron (Fig. 6A) or at the VR level (Fig. $6 B_{1}, B_{2}$ ) (see Materials and Methods), as well as by the inhibitory effect exerted on network output by the repetitive activation of some single cells $(n=3)$ (Fig. 6C). Neurons in the spinal gray were impaled and allowed to recover from the initial depolarization. Only cells in which the resting membrane potential stabilized below $-67 \mathrm{mV}$ were used $(-76.7 \pm 5.1 \mathrm{mV})$. Input resistance $\left(R_{\mathrm{in}}\right)$ and principal time constant $\left(\tau_{0}\right)$ were estimated by fitting a single exponential to the slower part of the voltage response to a negative current step (50-300 pA). The wide range of $R_{\text {in }}$ observed (2-236 M $\Omega$ ) correlated with marked differences in cell size as determined subjectively by how difficult it was to obtain and maintain a stable membrane potential. $\tau_{0}$, which also varied considerably $(6-111$ $\mathrm{ms})$, had a positive and rather steep linear correlation with $R_{\mathrm{in}}\left(r^{2}\right.$ $=0.86$; regression coefficient, 0.41 ) that remained when the common logarithm of both variables was used to compensate for the uneven distribution of data points (Fig. 6D) $\left(r^{2}=0.64\right)$. Previously reported by Buchanan (1993), this positive correlation is not an obvious finding because $\tau_{0}$ should not vary with cell size if resistivity stays constant. Thus, the correlation suggests a higher membrane resistivity and/or a less uniform somatodendritic distribution of membrane resistivity in the smaller neurons (Koch, 1999; London et al., 1999). We cannot exclude, however, that in the small cells with the highest values of $\tau_{0}$, there could be an additional effect of a closing down of voltage-dependent ion channels by the hyperpolarizing test pulse. $R_{\text {in }}$ for motor interneurons and EINs was in the range previously reported (Fig. 6E) (cf. Buchanan, 1993). Inhibitory and unidentified neurons had wide-ranging $R_{\text {in }}$ values.
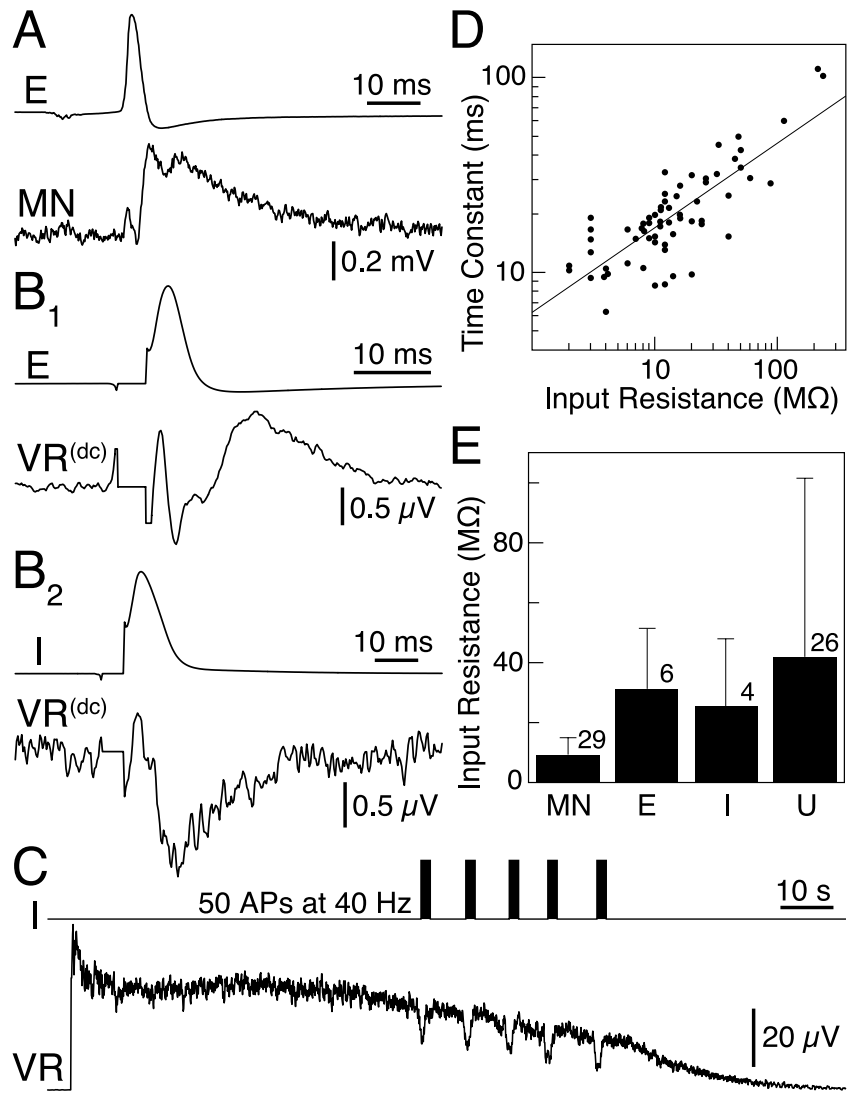

Figure 6. Identification and main parameters of neurons recorded intracellularly at the soma. $A$, An EIN (E; top trace) identified by paired recording with a postsynaptic MN is shown. The action potential in E elicits an EPSP in the MN (bottom trace). $B_{1}, B_{2}$, Excitatory and inhibitory (I) premotor interneurons were here identified by $D C$ recording of their synaptic potentials at the VR (Brink et al., 1981; Butt and Kiehn, 2003). C, Inhibitory interneurons were, in some cases, powerful enough to reduce efferent VR activity when induced to fire at high frequency. APs, Action potentials. D, A wide range of principal time constants and input resistances was observed among the neurons recorded. A linear relationship was apparent between their logtransformed values $\left(r^{2}=0.64\right)$, as previously reported by Buchanan (1993). $E$, Range of input resistances within the different neuronal classes. U, Unidentified neuron. Error bars indicate SD, and the numbers above each bar give the number of cells in each class.

\section{A subpopulation of spinal neurons are recruited during a bout}

What type of synaptic input do single neurons receive, and how is it integrated to produce the firing pattern described above? When the hemicord was electrically stimulated eliciting a locomotor bout, most cells exhibited a long-lasting depolarization $(n=59$; range, $0.3-23.2 \mathrm{mV}$ ) (Fig. $7 A_{1}, A_{2}, C$ ), on top of which locomotorrelated oscillations appeared. This depolarization was sufficient to evoke sustained spiking in 28 cases (Fig. $7 B$ ) and occasional action potentials in another 10 cases $\left(\mathrm{Fig} .7 A_{1}\right)$. The change in membrane potential after stimulation was very fast $(<300 \mathrm{~ms})$, unless stimulus intensity was reduced to threshold, thus evoking a gradual buildup of network activity (data not shown). The depolarization (and spiking frequency) decreased progressively during the bout, in parallel with the reduction in efferent VR activity. Strong negative or positive current pulses (1-2 s) injected in the cell soma were not able to potentiate or prematurely terminate the depolarization ( $n=5$; data not shown). A few neurons instead exhibited a hyperpolarization $(n=6$; range, -0.2 to $-4.3 \mathrm{mV}$ ) (Fig. $7 \mathrm{~B}, \mathrm{C}$ ), a phenomenon also observed during fictive swimming in the intact cord (Brodin and Grillner, $1986 b)$. The dual recording of Figure $7 B$ exemplifies how differ- 
ent cells from the same preparation displayed different behavior during a locomotor bout: strong depolarization with spiking in a MN and slight hyperpolarization in an unidentified neuron. It therefore appears that there is not an unspecific neuronal activation, but the recruitment of a well delimited network. The peak change in membrane potential after electrical stimulation is summarized, by neuron type, in Figure $7 D$. MNs received a strong excitation from the premotor network ( $16.1 \pm 3.9 \mathrm{mV}$; range, $8.0-22.5 \mathrm{mV})$, and most of them fired action potentials (25 of 29). The EINs received, on average, a mild net excitatory input $(2.8 \pm 4.0 \mathrm{mV}$; range, -4.3 to $6.1 \mathrm{mV}$ ), as did the inhibitory interneurons $(2.5 \pm 3.1 \mathrm{mV}$; range, -0.5 to $6.1 \mathrm{mV})$. The firing threshold was not reached in any of these cells. The larger group of unidentified neurons showed a wide range of responses $(7.8 \pm 7.4 \mathrm{mV}$; range, -3.9 to $23.2 \mathrm{mV}$ ) including at least some firing in 13 cases. We subdivided this population in two categories according to $R_{\text {in }}$, those $<26 \mathrm{M} \Omega$ and those $>40 \mathrm{M} \Omega$, the second most certainly not containing MNs (compare Fig. 6E) (Teravainen and Rovainen, 1971; Buchanan, 1993). Both populations had large cell-to-cell response variability (Fig. 7D). The lack of spiking in the EINs might be attributable to a combination of microelectrode-induced shunting and a sample bias toward larger somata, which might receive less excitation. In this latter case, the EINs that we recorded from might not have been part of the active subpopulation generating the rhythm. In one EIN, we observed a hyperpolarization $(-4.3 \mathrm{mV})$, a finding compatible with an additional subdivision of these cells.

\section{Fast membrane potential oscillations are visible below the} firing threshold

We looked for evidence of a structure in synaptic input by analyzing the membrane potential fluctuations occurring during a locomotor bout in those neurons that did not fire, or that fired only transiently $(n=43)$. All of these neurons showed an increase in the amplitude of their membrane potential fluctuations from quiescence (average RMS, $0.23 \pm 0.12 \mathrm{mV}$ ) to the locomotor bouts (average RMS, $0.92 \pm 0.33 \mathrm{mV} ; p<0.001$; Wilcoxon matched-pairs test) (Fig. $8 C$ ), during which they displayed fast oscillations (Fig. $8 \mathrm{~A}_{1}$ and $\mathrm{B}_{1}$ ). Note that the peak-to-peak amplitude of the oscillations will be considerably larger than their RMS value (which is equivalent to only $1 \mathrm{SD}$ ). These oscillations were rather variable in amplitude or duration from cycle to cycle, and individual synaptic potentials could often be resolved. Crosscorrelation analysis between the intracellular and VR records within one segment (see Materials and Methods) revealed a significant central peak ( $>4$ SEs) in 40 neurons (Fig. $8 A_{2}, B_{2}$ ) (correlation coefficient, $0.25 \pm 0.12$ ). The preferred phase of the oscillations with respect to the VR bursts was measured on the cross-correlogram by the positive or negative time delay of the central peak, divided by the cycle period and multiplied by $360^{\circ}$. In this context, a phase of $0^{\circ}$ would imply that the depolarizing part of the oscillations is centered (on average) on the middle of a
VR burst (Fig. $8 D$, inset). Values for all cells are summarized in Figure $8 \mathrm{D}$. In most cases, the membrane depolarization had a tendency to follow the VR burst (i.e., preferred phase $>0^{\circ}$ ) with a short phase lag $\left(25 \pm 18^{\circ}\right.$; range, -20 to $\left.50^{\circ}\right)$. No neuron was found that had membrane potential oscillations in antiphase with VR bursting (i.e., preferred phase less than $-90^{\circ}$ or $>90^{\circ}$ ) (Fig. $8 D)$. Oscillations in MNs consistently showed a phase delay (31 $\pm 7^{\circ} ; n=14 ; p<0.001$; Wilcoxon signed rank test) (Fig. $8 E$ ). Because MNs should spike, on average, with a preferred phase of $0^{\circ}$ (VR bursts are composed of these spikes) (Fig. 9B), one may conclude that these spikes occur early in each oscillation, as the rising membrane potential crosses the firing threshold. This difference between the preferred phase of firing and that of the oscillations was confirmed in five MNs, in which both values could be measured during the same recording. In all cases, the latter $\left(29 \pm 9^{\circ}\right)$ was delayed to the former $\left(4 \pm 4^{\circ} ; p=0.06\right.$; Wilcoxon matched-pairs test). EINs and unidentified neurons had a wider range of preferred oscillation phases $\left[14 \pm 18^{\circ}(n=\right.$ $6)$ and $21 \pm 22^{\circ}(n=17)$, respectively] but generally phase advanced MNs $(p<0.05$ and $p=0.23$, respectively; WilcoxonMann-Whitney test). The three inhibitory interneurons were somewhat more delayed ( $42 \pm 4^{\circ} ; n=3 ; p<0.05$ ).

Intracellularly recorded neurons fire one spike per locomotor cycle

Cross-correlation analysis between action potential events and the VR record revealed a significant central peak ( $>4$ SEs) in 28 of 29 neurons (of all classes) that spiked sufficiently to be analyzed. Their preferred phase of firing with respect to the VR bursts was measured on the cross-correlogram, similarly to the oscillations. Thus, a phase of $0^{\circ}$ would imply that the action potentials occur (on average) in the middle of a VR burst (Fig. 9A, inset). The 


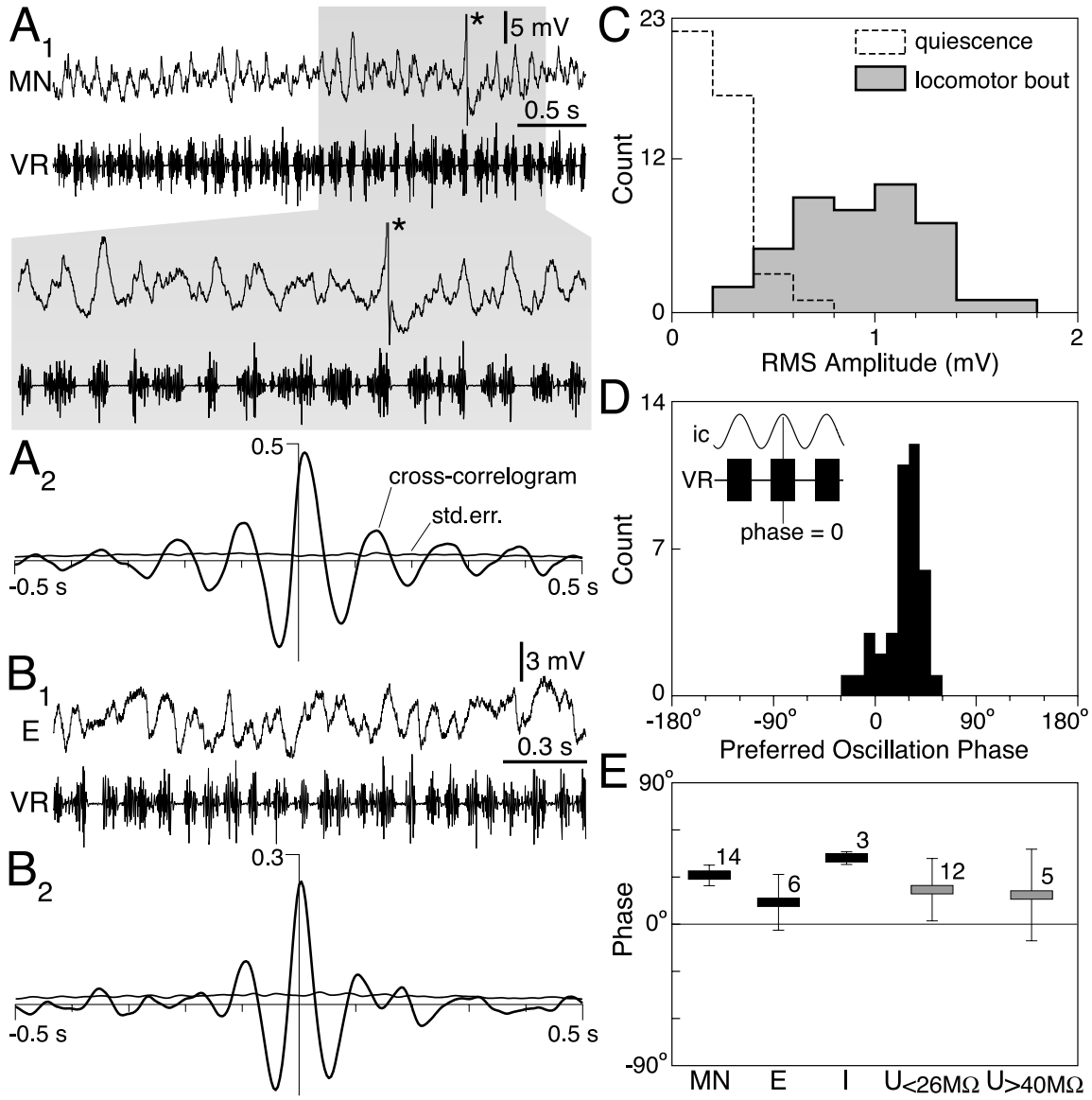

Figure 8. Neurons display fast membrane potential oscillations in phase with VR bursting. $A_{1}, B_{1}$, Magnified sections of recording in a MN and EIN (E) during their depolarized state in a bout (each asterisk marks a truncated spike). $A_{2}, B_{2}$, Crosscorrelograms of the intracellular and the rectified-smoothed VR records, together with their SE levels. C, Distribution across neurons of the RMS amplitude of membrane potential fluctuations in the quiescent preparation (empty, dashed histogram) and during the locomotor bouts (filled, continuous histogram). D, Distribution of the preferred phase of the oscillations with respect to the VR bursts. The inset shows schematically what is meant by a phase of zero. Note that amplitude and phase analysis in C and D could be performed only for neurons that did not fire continuously during the bouts. E, Preferred oscillation phase by neuronal class. Error bars indicate SD, and the numbers above each bar give the number of cells in each class. std.err., SE; $U$, unidentified neuron.

preferred firing phases of all 28 active neurons were distributed at approximately zero (average not significantly different from zero with $p=0.26$; Wilcoxon signed rank test), and none was tuned to fire in antiphase $\left(0 \pm 21^{\circ}\right.$; range, -68 to $\left.45^{\circ}\right)$ (Fig. $\left.9 A\right)$. MNs showed a relatively narrow distribution of preferred phases $\left(-1 \pm 15^{\circ} ; n=18\right)$ (Fig. $\left.9 B\right)$ but clearly that a certain degree of cycle-to-cycle jitter in their phase of firing contributes to the duration of VR bursts. This spread in preferred firing phases, as well as the cycle-to-cycle jitter, are likely to depend on differences in the set of excitatory inputs that converge onto each $\mathrm{MN}$ (Buchanan and Kasicki, 1999). Under these conditions, the excitatory and inhibitory interneurons recorded here did not fire action potentials, whereas the unidentified neurons had a broad distribution of preferred phases $\left(1 \pm 30^{\circ} ; n=10\right)$. The pattern of firing of all cells was rather regular $\left(\mathrm{CV}_{\mathrm{ISI}}=0.23 \pm 0.06\right.$, measured between 10 and $20 \mathrm{~s}$ from bout start) and was analyzed in the same way as the axonal recordings of MNs and ipsilaterally projecting interneurons. Histograms were built counting the occurrence of ISIs normalized to the average cycle period of VR bursting. MNs (Fig. 9C), as well as large (Fig. 9E) and small unidentified neurons (Fig. 9F), fired one spike for every locomotor cycle or less. Data were taken from the first $66 \%$ of the spike discharge of the neurons, when activity was higher. In the re- maining $34 \%$ of the spike discharge, essentially no multiple firings during a cycle were seen $(0.47 \%$ of normalized ISI below $0.5)$. Although the EINs we recorded from did not reach the firing threshold spontaneously, spiking was evoked in three of them by injecting long pulses (2-3 s) of tonic current $(<0.3 \mathrm{nA})$ right after the start of the locomotor bout. The resulting activity was in line with our general conclusion that, in the electrically activated hemicord, spinal neurons fire one spike for every locomotor cycle or less (Fig. 9D).

\section{5-HT increases the locomotor burst frequency}

On the basis of the neuronal firing pattern observed in the hemicord, any manipulation affecting the firing rate of EINs would be expected to also influence the frequency of bursting at the VRs. In a recent study, Biró et al. (2003) showed that the afterhyperpolarization in EINs, as in MNs (Wallén et al., 1989), is reduced by the neuromodulator 5-HT. 5-HT should thus increase the firing rate of individual EINs in response to a given input. We perfused the hemicord with $1 \mu \mathrm{M} 5$-HT $(n=5)$ and measured its effects on network output (Fig. 10A shows a plot of efferent activity vs burst frequency for one preparation). We found that the peak burst frequency during a bout was significantly increased in each preparation $(p<0.001$; Wilcoxon-Mann-Whitney test) from an average of $12 \pm 2 \mathrm{~Hz}$ to $21 \pm 3 \mathrm{~Hz}$ (Fig. $10 \mathrm{~A}$, rightmost data points). In the intact spinal cord, 5-HT instead slows down the locomotor rate (Harris-Warrick and Cohen, 1985) by facilitating crossed inhibition and by prolonging bursts in individual neurons via a reduction of the afterhyperpolarization summation leading to decreased spike frequency adaptation (Kozlov et al., 2001). In the hemicord, in contrast, crossed inhibition is absent and neurons fire one spike per cycle. The effect of 5-HT in the hemicord can thus be easily interpreted in the context of the model of Figure $10 B$ [see figure legend and Discussion; see also simulations in Tegnér (1997)]. In four of five preparations, we also observed a significant decrease in efferent activity at any given burst frequency $(p<0.05$; Wilcoxon matched-pairs test on data binned and averaged by frequency interval) of $-27 \pm 13 \%$ (Fig. 10 A, downward shift in data points). This suggests a reduction in the number of active MNs in 5-HT compared with control conditions (compare Fig. $1 F$ ) and could be explained by the depression known to occur at the EIN-MN synapse in 5-HT (Parker and Grillner, 1999; Biró et al., 2003). In parallel with the increased burst rates, rhythmic quality at the beginning of the bouts diminished in all preparations $(p<$ 0.05; Wilcoxon-Mann-Whitney test).

\section{Discussion}

This work was based on our recent finding that the lamprey hemicord maintains the capability of expressing the swim 

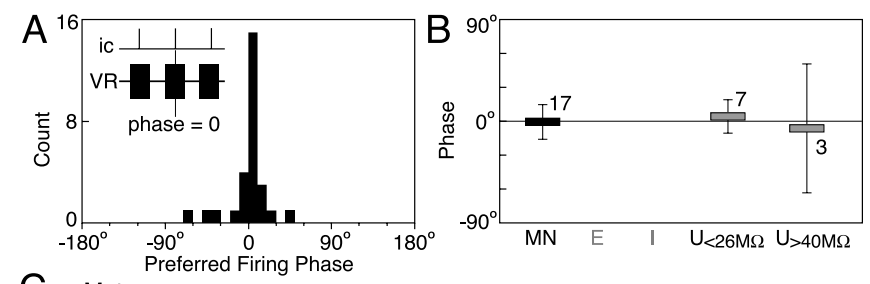

C - Motoneurons

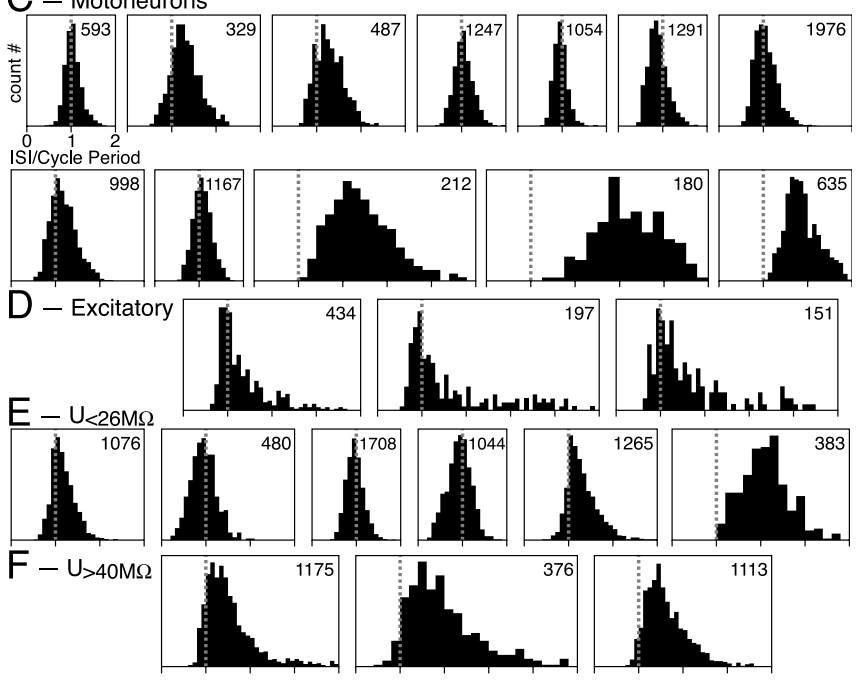

Figure 9. Neurons reaching firing threshold during a bout fire one action potential for every locomotor cycle or less. $A$, Distribution of preferred firing phases in all spiking neurons recorded. The inset shows schematically what is meant by a phase of zero. $B$, Preferred firing phase by neuronal class. [None of the excitatory $(E)$ or inhibitory (I) neurons we recorded from fired sufficiently to be analyzed.] The numbers above each bar give the number of cells in each class. $U$, Unidentified neuron. $C-F$, Histograms of the ISIs normalized to the average cycle period of VR bursting (see Figs. 4, 5) for all spiking neurons recorded, subdivided by class. The EINs were induced to fire by tonic current injection during the bout. The number at the top right of each panel indicates the number of ISIs used in generating each histogram.

rhythm, despite the lack of phasic inhibitory input from the contralateral side (Cangiano and Grillner, 2003). Here, we investigated the functioning of the unilateral network at medium-fast rates of swimming, by activating it with brief electrical stimuli. We found evidence for recurrent excitation within the hemicord, likely to be mediated by EINs. Fast glutamatergic transmission is required for the long bouts of burst activity to occur. Once a bout is evoked, an activity-dependent mechanism will depress excitability, thereby progressively reducing the burst rate until the bout terminates. In quiescence, the hemicord gradually recovers excitability. Individual interneurons and MNs tend to fire one spike for every locomotor cycle at the peak of network activity (multiple firings during a cycle were extremely rare), whereas they may skip one or more cycles when activity diminishes. The frequency of VR bursting thus corresponds to the average ISI of the most active neurons.

As mentioned in Results, the integrative properties of a neuron could change because of the damage inflicted by an intracellular microelectrode. We thus performed extracellular recordings to complement the intracellular ones. The same neuronal firing pattern was observed. This greatly increased our confidence in these data, supporting recent technical considerations in Xenopus (Li et al., 2004). With regard to our intracellular measurements of membrane depolarization and oscillation/firing phase, we cannot exclude some influence of impalement damage.

In the intact spinal cord, even strong electrical stimuli do not
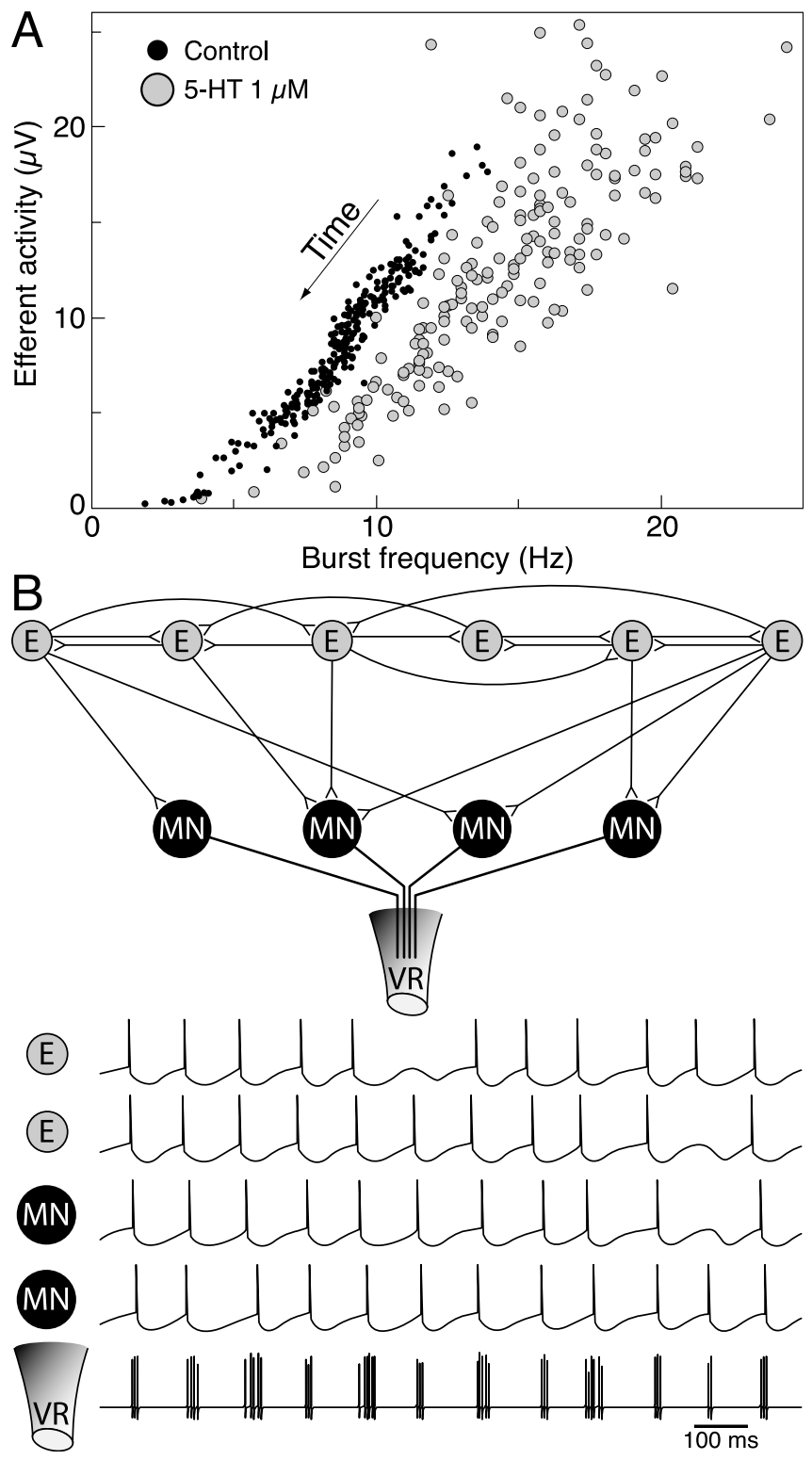

Figure 10. The network effect of 5-HT and a model for rhythm generation in the hemicord. $A$, Plot showing the relationship between efferent activity and burst frequency in control and during perfusion of the neuromodulator $5-\mathrm{HT}(1 \mu \mathrm{m})$. Peak burst frequencies strongly increased. Efferent activity at any given frequency was reduced, thus suggesting fewer active MNs (compare Fig. 1F). B, The simplest model network that could account for the fast rhythmic bursting expressed by the hemicord, based on the observed firing pattern of single neurons and the effect of 5-HT. Only one hemisegment is shown, with the circles representing groups of neurons. EINs (E) form an interconnected network able to sustain repetitive firing by positive feedback, mediated by both fast ionotropic and slow metabotropic synaptic transmission. The afterhyperpolarization that follows an action potential will regulate the ISI in each neuron. Fast synaptic interactions promote a certain degree of synchronization across the population: as a new spike occurs after a period of hyperpolarization, it will excite other EINs and stimulate their firing. Phasic excitation is relayed to the MNs, which thus also tend to fire in synchrony with the EINs. At each cycle, the individual action potentials from many MNs converge in the VR, where a burst is recorded. In this model, 5 -HT would increase peak burst rates by reducing the spike afterhyperpolarization (Biró et al., 2003) and diminish the number of active MNs by depressing the E to MN synapse.

elicit more than a few seconds of VR activity (Cohen and Wallén, 1980). This activity tends to be asymmetrical, because small differences in the effective stimulation between the two sides are amplified by crossed inhibition (Fagerstedt et al., 2000). Interest- 
ingly, if only one side of the spinal cord is stimulated, fast rhythmic bursts in the same frequency range as the hemicord can be observed in the ipsilateral VRs, whereas the contralateral side is inhibited (our unpublished observation). The short duration of VR activity in response to electrical stimulation was correctly ascribed to the strong crossed inhibition in the locomotor network (Fagerstedt et al., 2000). This prevents buildup of activity in the absence of the background excitatory input normally provided by the brainstem. The hemicord, in contrast, given that the initial electrical stimulus reaches a threshold intensity, is able to self-maintain activity up to several minutes. This is strong evidence that EINs form a recurrently interconnected network on each side of the spinal cord. The synaptic connections of EINs onto other EINs has recently been demonstrated by dual intracellular recording but was never found to be mutual (Parker and Grillner, 2000). If this is the norm, these cells must then form a sparsely interconnected network such that excitation may feed back onto any given neuron via several other interposed EINs. That the core of individual burst generators is attributable to a network of interacting excitatory neurons may be a general organizational feature, because it does not apply only to locomotor networks but also to respiration (pre-Botzinger complex) and saccadic burst generators as recently discussed (Kiehn et al., 2004).

Once a bout is evoked, an activity-dependent mechanism acting at the premotoneuronal level progressively reduces excitability, eventually bringing the hemicord back to quiescence. As to the nature and site of action of this depression, it could involve either diminution of a network resource (e.g., a neurotransmitter as glutamate) or the buildup of a limiting factor. The first alternative (a depression of synaptic efficacy) is compatible with our observation that a partial blockade of glutamatergic transmission and the activity-dependent decrease in excitability have a similar effect on the network (Fig. 2, bottom panels). Unfortunately, EIN-EIN synapses have only been tested for brief trains of 20 EPSPs (Parker and Grillner, 2000). In the chick embryo, the spinal cord exhibits spontaneous bursts that are thought to terminate as a consequence of slow synaptic depression (Tabak et al., 2000). Among the second type of mechanisms, the electrogenic $\mathrm{Na} / \mathrm{K}$ pump could be considered: intense network activity will cause $\mathrm{Na}^{+}$entry that might lead to enhanced pump activity. This would produce a hyperpolarization of spinal neurons and thus a decrease in their firing rate. This mechanism was proposed to influence the duration of bursts in the disinhibited neonatal rat spinal cord (Ballerini et al., 1997) and embryonic rat cultures of spinal neurons (Darbon et al., 2003). Moreover, an activitydependent buildup of adenosine as a consequence of neuronal activity serves as a main factor in the termination of swimming bouts in the Xenopus embryo (Dale and Gilday, 1996; Brown and Dale, 2000).

Another important question is what mechanisms underlie the fast rhythmic bursting expressed by the hemicord at its VRs. Our data on the firing pattern of interneurons and MNs restrict the possibilities. Because each VR burst can be as short as a few tens of milliseconds, its termination is unlikely to depend on fast synaptic depression between EINs. Moreover, multiple spikes per cycle were extremely rare. For the same reason, spike frequency adaptation, which is thought instead to come into play at lower locomotor burst rates (Kotaleski et al., 1999; Grillner et al., 2001), may be excluded. The simplest model that could account for the generation of rhythmic bursting by the hemicord is that outlined in Figure $10 \mathrm{~B}$. EINs would form mutual connections sufficient to maintain sustained firing in the premotor network and in MNs.
This mutual excitation may rely not only on fast synaptic transmission but also on slower metabotropic components (Krieger et al., 1998; Kettunen et al., 2003). The EINs would fire at a rate dictated by the level of this background excitation, as well as their specific frequency response characteristics. Fast synaptic excitation would act to promote synchronization within the EIN population and thus phasically modulated excitation to the EINs themselves and to MNs. Individual MNs would fire once in every cycle, while their population activity is recorded as a burst at the VR. In this model, the mechanism regulating burst frequency at the VRs is the same that controls the ISI in individual neurons. In lamprey MNs, this role is played mainly by the slow afterhyperpolarization (Hill et al., 1992; Meer and Buchanan, 1992; El Manira et al., 1994; Cangiano et al., 2002) and also in EINs, although studied to a lesser extent (Buchanan et al., 1989; Biró et al., 2003). The increase in the peak burst frequencies caused by 5 -HT further supports this model. In addition, if our and Buchanan's observation that small spinal neurons have a significantly longer time constant applies also to small EINs, this might complement hyperpolarizing conductances in limiting spike frequency during high-frequency firing. Our model does not exclude the contribution of transmitters and modulators other than glutamate, for example, in regulating network excitability. Moreover, neuronal synchronization could be aided in part by dendro-dendritic gap junctions.

Fast rhythmic bursting can be evoked in the hemicord not only by electrical stimulation but also pharmacologically by perfusing D-glutamate or NMDA (Cangiano and Grillner, 2003). In the same study, we showed that this pattern is directly related to fictive locomotion in the intact spinal cord. Before our development of the hemispinal cord preparation, a similar fast rhythm had been observed in the intact cord during a blockade of glycinergic inhibition with strychnine (Cohen and HarrisWarrick, 1984). The results reported here on the firing pattern of single neurons should also extend to these other situations. It may be noted that the hemicord can also display slow VR burst activity within the locomotor range in NMDA, relying on the voltage-dependent plateau properties of NMDA receptors (Cangiano and Grillner, 2003), a pattern that was not studied in this report.

What can we infer about the operation of the locomotor CPG from these experiments on the unilateral network deprived of phasic inhibitory input from the contralateral side? The excitability of the network is apparently high, and it can sustain rhythmic activity in the mid to upper frequency range of locomotion. Several past studies have looked at the firing pattern of premotorneurons and $\mathrm{MNs}$ in the intact cord during fictive swimming (Buchanan and Cohen, 1982; Russell and Wallén, 1983; Wallén et al., 1985; Buchanan et al., 1989; Buchanan and Kasicki, 1995). These reported that active neurons may fire several action potentials (i.e., a short burst) during each locomotor cycle. Compared with our data in the electrically activated hemicord, it thus appears that crossed inhibition, which lowers the endogenous burst frequency of the unilateral network (Cangiano and Grillner, 2003), also promotes a shift in the firing pattern of individual neurons from single to multiple action potentials per cycle. This shift might be a consequence of the lower burst rates in the intact cord but could also be encouraged by postinhibitory rebound (Grillner et al., 2001). With lower burst rates and a different neuronal firing pattern, other cellular mechanisms will come into play during locomotion. 


\section{References}

Aiken SP, Kuenzi FM, Dale N (2003) Xenopus embryonic spinal neurons recorded in situ with patch-clamp electrodes - conditional oscillators after all? Eur J Neurosci 18:333-343.

Alford S, Grillner S (1990) CNQX and DNQX block non-NMDA synaptic transmission but not NMDA-evoked locomotion in lamprey spinal cord. Brain Res 506:297-302.

Ballerini L, Bracci E, Nistri A (1997) Pharmacological block of the electrogenic sodium pump disrupts rhythmic bursting induced by strychnine and bicuculline in the neonatal rat spinal cord. J Neurophysiol 77:17-23.

Biró Z, Hill RH, Grillner S (2003) 5-HT modulation of excitatory interneurons in the lamprey spinal cord. Soc Neurosci Abstr 29:188.7.

Brink E, Jankowska E, McCrea D, Skoog B (1981) Use of sucrose gap for recording postsynaptic population potentials by single interneurones in spinal motoneurones. Brain Res 223:165-169.

Brodin L, Grillner S (1986a) Effects of magnesium on fictive locomotion induced by activation of $N$-methyl-D-aspartate (NMDA) receptors in the lamprey spinal cord in vitro. Brain Res 380:244-252.

Brodin L, Grillner S (1986b) Tonic inhibition of a new type of spinal interneurone during fictive locomotion in the lamprey. Acta Physiol Scand 128:327-329.

Brown P, Dale N (2000) Adenosine A1 receptors modulate high voltageactivated $\mathrm{Ca}^{2+}$ currents and motor pattern generation in the Xenopus embryo. J Physiol (Lond) 525:655-667.

Buchanan JT (1993) Electrophysiological properties of identified classes of lamprey spinal neurons. J Neurophysiol 70:2313-2325.

Buchanan JT, Cohen AH (1982) Activities of identified interneurons, motoneurons, and muscle fibers during fictive swimming in the lamprey and effects of reticulospinal and dorsal cell stimulation. J Neurophysiol 47:948-960.

Buchanan JT, Grillner S (1987) Newly identified "glutamate interneurons" and their role in locomotion in the lamprey spinal cord. Science 236:312-314.

Buchanan JT, Grillner S (1988) A new class of small inhibitory interneurons in the lamprey spinal cord. Brain Res 438:404-407.

Buchanan JT, Kasicki S (1995) Activities of spinal neurons during brain stem-dependent fictive swimming in lamprey. J Neurophysiol 73: $80-87$.

Buchanan JT, Kasicki S (1999) Segmental distribution of common synaptic inputs to spinal motoneurons during fictive swimming in the lamprey. J Neurophysiol 82:1156-1163.

Buchanan JT, Grillner S, Cullheim S, Risling M (1989) Identification of excitatory interneurons contributing to generation of locomotion in lamprey: structure, pharmacology, and function. J Neurophysiol 62: 59-69.

Butt SJB, Kiehn O (2003) Functional identification of interneurons responsible for left-right coordination of hindlimbs in mammals. Neuron 38:953-963.

Cangiano L, Grillner S (2003) Fast and slow locomotor burst generation in the hemispinal cord of the lamprey. J Neurophysiol 89:2931-2942.

Cangiano L, Wallén P, Grillner S (2002) Role of apamin-sensitive k(ca) channels for reticulospinal synaptic transmission to motoneuron and for the afterhyperpolarization. J Neurophysiol 88:289-299.

Cohen AH, Harris-Warrick R (1984) Strychnine eliminates alternating motor output during fictive locomotion in the lamprey. Brain Res 293:164-167.

Cohen AH, Wallén P (1980) The neuronal correlate of locomotion in fish. Exp Brain Res 41:11-18.

Dale N (1986) Excitatory synaptic drive for swimming mediated by amino acid receptors in the lamprey. J Neurosci 6:2662-2675.

Dale N (1995) Experimentally derived model for the locomotor pattern generator in the Xenopus embryo. J Physiol (Lond) 489:489-510.

Dale N, Gilday D (1996) Regulation of rhythmic movements by purinergic neurotransmitters in frog embryos. Nature 383:259-263.

Darbon P, Tscherter A, Yvon C, Streit J (2003) Role of the electrogenic Na/K pump in disinhibition-induced bursting in cultured spinal networks. J Neurophysiol 90:3119-3129.

Earhart GM, Stein PS (2000) Step, swim, and scratch motor patterns in the turtle. J Neurophysiol 84:2181-2190.

El Manira A, Tegnér J, Grillner S (1994) Calcium-dependent potassium channels play a critical role for burst termination in the locomotor network in lamprey. J Neurophysiol 72:1852-1861.
Fagerstedt P, Zelenin PV, Deliagina TG, Orlovsky GN, Grillner S (2000) Crossed reciprocal inhibition evoked by electrical stimulation of the lamprey spinal cord. Exp Brain Res 134:147-154.

Grillner S (2003) The motor infrastructure: from ion channels to neuronal networks. Nat Rev Neurosci 4:573-586.

Grillner S, Wallén P (1980) Does the central pattern generation for locomotion in lamprey depend on glycine inhibition? Acta Physiol Scand 110:103-105.

Grillner S, Cangiano L, Hu GY, Thompson R, Hill R, Wallén P (2000) The intrinsic function of a motor system-from ion channels to networks and behavior. Brain Res 886:224-236.

Grillner S, Wallén P, Hill R, Cangiano L, El Manira A (2001) Ion channels of importance for the locomotor pattern generation in the lamprey brainstem-spinal cord. J Physiol (Lond) 533:23-30.

Harris-Warrick RM, Cohen AH (1985) Serotonin modulates the central pattern generator for locomotion in the isolated lamprey spinal cord. J Exp Biol 116:27-46.

Hill RH, Matsushima T, Shotland J, Grillner S (1992) Apamin blocks the slow AHP in lamprey and delays termination of locomotor bursts. NeuroReport 3:943-945.

Kettunen P, Hess D, El Manira A (2003) MGluR1, but not mGluR5, mediates depolarization of spinal cord neurons by blocking a leak current. J Neurophysiol 90:2341-2348.

Kiehn O, Butt SJ (2003) Physiological, anatomical and genetic identification of CPG neurons in the developing mammalian spinal cord. Prog Neurobiol 70:347-361.

Kiehn O, Büschges A, Duch C, Grillner S, Isa T, Lansner A, Pflüger H-J, Richter DW, Sillar KT, Smith J, Sparks DL (2005) Microcircuits in the motor system. In: Microcircuits: the interface between neurons and global brain function. Dahlem Workshop Report 93 (Grillner S, Graybiel A, eds). Cambridge, MA: MIT, in press.

Koch C (1999) Biophysics of computation: information processing in single neurons, pp 75-77. New York: Oxford UP.

Kotaleski JH, Lansner A, Grillner S (1999) Neural mechanisms potentially contributing to the intersegmental phase lag in lamprey. II. Hemisegmental oscillations produced by mutually coupled excitatory neurons. Biol Cybern 81:299-315.

Kozlov A, Kotaleski JH, Aurell E, Grillner S, Lansner A (2001) Modeling of substance $\mathrm{P}$ and 5-HT induced synaptic plasticity in the lamprey spinal CPG: consequences for network pattern generation. J Comput Neurosci 11:183-200.

Kremer E, Lev-Tov A (1997) Localization of the spinal network associated with generation of hindlimb locomotion in the neonatal rat and organization of its transverse coupling system. J Neurophysiol 77:1155-1170.

Krieger P, Grillner S, El Manira A (1998) Endogenous activation of metabotropic glutamate receptors contributes to burst frequency regulation in the lamprey locomotor network. Eur J Neurosci 10:3333-3342.

Li W, Soffe SR, Roberts A (2004) A direct comparison of whole-cell patch and sharp electrodes by simultaneous recording from single spinal neurons in frog tadpoles. J Neurophysiol 92:380-386.

London M, Meunier C, Segev I (1999) Signal transfer in passive dendrites with nonuniform membrane conductance. J Neurosci 19: 8219-8233.

McPherson DR, Buchanan JT, Kasicki S (1994) Effects of strychnine on fictive swimming in the lamprey: evidence for glycinergic inhibition, discrepancies with model predictions, and novel modulatory rhythms. J Comp Physiol [A] 175:311-321.

Meer DP, Buchanan J (1992) Apamin reduces the late afterhyperpolarization of lamprey spinal neurons, with little effect on fictive swimming. Neurosci Lett 143:1-4.

Parker D, Grillner S (1999) Activity-dependent metaplasticity of inhibitory and excitatory synaptic transmission in the lamprey spinal cord locomotor network. J Neurosci 19:1647-1656.

Parker D, Grillner S (2000) The activity-dependent plasticity of segmental and intersegmental synaptic connections in the lamprey spinal cord. Eur J Neurosci 12:2135-2146.

Poon MLT (1980) Induction of swimming in lamprey by L-DOPA and amino acids. J Comp Physiol 136:337-344.

Roberts A, Perrins R (1995) Positive feedback as a general mechanism for sustaining rhythmic and non-rhythmic activity. J Physiol (Paris) 89:241-248. 
Roberts A, Soffe SR, Wolf ES, Yoshida M, Zhao FY (1998) Central circuits controlling locomotion in young frog tadpoles. Ann NY Acad Sci 860:19-34.

Rossignol S, Dubuc R (1994) Spinal pattern generation. Curr Opin Neurobiol 4:894-902.

Rovainen CM (1974) Synaptic interactions of identified nerve cells in the spinal cord of the sea lamprey. J Comp Neurol 154:189-206.

Rovainen CM, Dill DA (1984) Counts of axons in electron microscopic sections of ventral roots in lamprey. J Comp Neurol 225:433-440.

Russell DF, Wallén P (1983) On the control of myotomal motoneurones during "fictive swimming" in the lamprey spinal cord in vitro. Acta Physiol Scand 117:161-170.

Sillar KT, McLean DL, Fischer H, Merrywest SD (2002) Fast inhibitory synapses: targets for neuromodulation and development of vertebrate motor behaviour. Brain Res Brain Res Rev 40:130-140.
Tabak J, Senn W, O’Donovan J, Rinzel J (2000) Modeling of spontaneous activity in developing spinal cord using activity-dependent depression in an excitatory network. J Neurosci 20:3041-3056.

Tegnér J (1997) Modulation of cellular mechanisms in a spinal locomotor network: an experimental an computational study in lamprey. $\mathrm{PhD}$ thesis, Karolinska Institute.

Teravainen H, Rovainen CM (1971) Fast and slow motoneurons to body muscle of the sea lamprey. J Neurophysiol 34:990-998.

Wallén P, Grillner S, Feldman JL, Bergelt S (1985) Dorsal and ventral myotome motoneurons and their input during fictive locomotion in lamprey. J Neurosci 5:654-661.

Wallén P, Buchanan JT, Grillner S, Hill RH, Christenson J, Hökfelt T (1989) Effects of 5-hydroxytryptamine on the afterhyperpolarization, spike frequency regulation, and oscillatory membrane properties in lamprey spinal cord neurons. J Neurophysiol 61:759-768. 